\title{
Innovative Neonatal Laryngeal Mask for Airway Management in Piglets: a New Device for Potential use in Preterm and Term Neonates
}

\section{Ayoob Ali*}

Department of Pediatrics, Saint Louis University, United States of America

*Corresponding Author: Ayoob Ali, Department of Pediatrics, Saint Louis University, United States of America.
Received: June 25, 2021

Published: September 06, 2021

(C) All rights are reserved by Ayoob Ali.

\begin{abstract}
Background: Endotracheal intubation is an intricate process critical to maintaining airways during advanced neonatal resuscitation and administering surfactant to treat respiratory distress syndrome in premature infants. Although modern medical technology has created less strenuous means of maintaining airways during general anesthesia, these tools present evident limitations in many preterm and full-term babies. We examined the safety and efficacy of using a new device, the Neonatal Laryngeal Mask (NLM), to manage airways while inducing surgical plane anesthesia in piglets as well as facilitate tracheal catheterization to administer medication into the lungs.
\end{abstract}

Methods: Six piglets of domestic breed aged 3 - 7 days old and weighing 2300 - 3000 grams were used in this study. We monitored physiological parameters including, core body temperature, heart rate, end-tidal carbon dioxide $\left(\mathrm{EtCO}_{2}\right)$ and oxygen saturation $\left(\mathrm{SaO}_{2}\right)$ tracing before and after insertion of the NLM. Postmortem autopsy was conducted to examine the trachea and lungs to determine the extent of the spread of methylene blue as a measure of successful catheterization.

Results: There were no statistically significant differences present while maintaining normal physiological parameters before and after NLM insertion. This was noted when examining heart rate (U-value $14, p=0.416$ ), average core body temperatures (U-value 18 , $\mathrm{p}=0.468$ ), $\mathrm{SaO}_{2}$ levels (U-value 14, $\mathrm{p}=0.315$ ), and $\mathrm{EtCO}_{2}$ levels (U-value $11, \mathrm{p}=0.416$ ) in all piglets. Postmortem analysis confirmed the presence of methylene blue in the entire airway from the trachea to the lungs, reflecting proper catheter placement through the NLM into the lungs.

Conclusion: The new NLM device provides a safe and effective method for maintaining a patent airway and catheterizing a model system for pre-term and full-term infants. These findings are promising towards mitigating the current risks associated with caring for these populations and support further examination within human subjects as an investigational medical device.

Keywords: Neonatal Airway; Neonatal Laryngeal Mask; Piglet Anesthesia; Airway Management; Tracheal Catheterization

\section{Abbreviation}

NLM: Neonatal Laryngeal Mask

\section{Introduction}

Airway management constitutes a critical role in patient care. It is a standard practice to utilize endotracheal intubation for the induction and maintenance of airways during general anesthesia after intravenous localization to a surgical plane [1]. Moreover, endotracheal intubation is necessary to maintain airways during advanced neonatal resuscitation and administer surfactant to treat respiratory distress syndrome in premature infants [2]. Successful manual endotracheal intubation, however, is dependent on the presence of skilled personnel [3]. 
Modern developments in medical technology have created less strenuous means of maintaining airways during general anesthesia. Since its approval in 1999, the conventional Laryngeal Mask Airway ( $\left.\mathrm{LMA}^{\mathrm{TM}}\right)$, invented by Archibald Brain, represented the first non-invasive method of airway management [4-6]. However, the continued usage of the $\mathrm{LMA}^{\mathrm{TM}}$ presented evident restrictions in its application to various patient populations [7]. While the $\mathrm{LMA}^{\mathrm{TM}}$ had demonstrated efficacy in adults, children, and full-term newborns, it was unable to effectively manage airways in premature infants due to its respective size limitations $[8,9]$.

Thus we designed a new device-the Neonatal Laryngeal Mask (NLM)-that can both ventilate and administer medication into the lungs of premature infants. The NLM was designed after carefully reviewing computed tomography (CT) scan and magnetic resonance imaging (MRI) data for gestational-age infants and calculating estimated oropharyngeal dimensions. Unlike the conventional $\mathrm{LMA}^{\mathrm{TM}}$, the NLM was created using design specialized for premature and full-term babies.

Regarding its composition, the NLM is constituted by a disposable, silicone rubber tube connected to an elliptical-shaped mask characterized by a soft, inflatable cuff. In practice, this structure allows for the NLM to be inserted without the direct visualization of the glottis. It is, rather, administered (inserted) orally using the index finger and guided along the hard palate without the concurrent use of laryngoscopy or other instruments. The NLM cuff is inflated following successful placement, allowing for an induced occlusion of the esophagus and establishment of a patent airway from the trachea via openings in the mask lumen.

We developed the NLM in three sizes (NLM-00, NLM-0, and NLM-1) to accommodate usage in both premature as well as newborn babies from 27 weeks to 40 weeks of gestation. Likewise, these masks were designed for effective use in babies with a birth weight between 750 and 4000 grams. Given its specialized size, the NLM presents the potential to mitigate many of the aforementioned complications experienced while performing airway maintenance for premature babies during operation.

To investigate this novel approach for neonatal airway management, we examined the effects of using the NLM to manage airways while inducing piglets under general anesthesia by evaluating overall device efficacy and safety. We hypothesized that the unique form factor and lumen characteristics of the NLM contribute to a significant role in controlling the neonatal airway at ventilation levels comparable to current practices using manual endotracheal intubation. We also hypothesized that the design of the NLM would facilitate successful administration of surfactant under circumstances of respiratory distress.

\section{Materials and Methods}

\section{Materials}

A prototype NLM constructed as shown in figure 1.

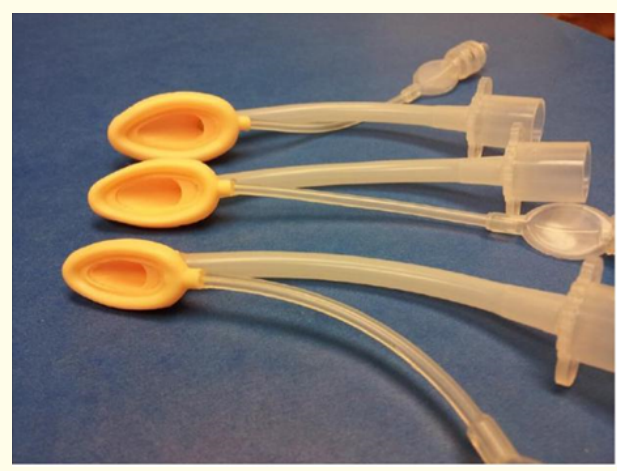

A

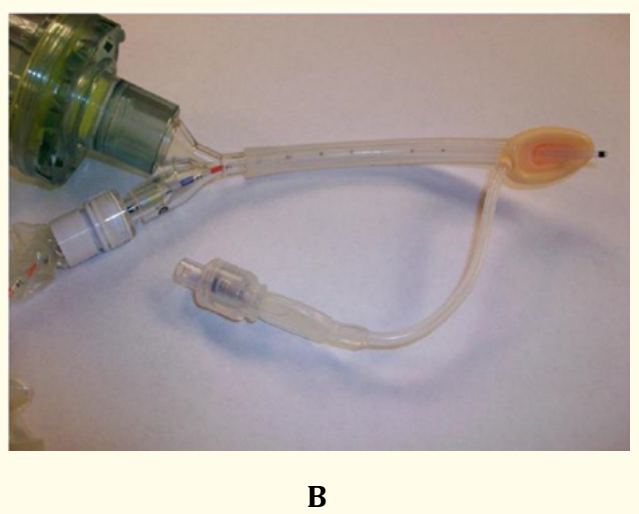

Figure 1: Structural and functional design of the NLM. The NLM consists as shown (A). A long tube with an inflatable donut-shaped cuff at one end lies over the larynx once it enters through the open mouth. This cuff is then inflated, allowing for the NLM to seals off from other structures that open into the pharynx (e.g. the esophagus). The other end of the NLM can then be attached to a resuscitation bag or mechanical ventilator using a v-adapter to provide mechanical ventilation (B). This device provides a more direct connection to the trachea than does a face mask and is less invasive than endotracheal tube intubation. 
Animal model

Animal studies were approved by the Institutional Animal Care Use Committee at Saint Louis University. Six piglets of domestic breed aged 3 - 7 days old weighing between 2300 - 3000 grams were used in this study. Piglet models were selected in order to provide useful and necessary information about the feasibility and safety of the NLM, due to the close resemblance of anatomical features with neonates.

\section{Study design}

This study was designed to evaluate the efficacy and safety of the NLM to control the airways of piglets during anesthesia and facilitate the administration of surfactant into the lungs. To assess overall efficacy, we examined parameters of resuscitation such as heart rate, oxygen saturations levels $\left(\mathrm{SaO}_{2}\right)$, end-tidal carbon dioxide $\left(\mathrm{EtCO}_{2}\right)$, and core body temperature before and after insertion of NLM as well during anesthesia administration while piglets breathing either spontaneously or via hand bagging. To assess safety, defined as complications or unwanted effects of the device, we monitored the number of attempts of successful and failed insertions of the NLM, events of bradycardia, and hypoxemia.

Piglets were initially placed in an acrylic chamber connected to an oxygen supply, and a surgical plane of anesthesia was induced by the academic veterinary anesthetist using 5\% isoflurane in 4 $\mathrm{L} /$ min oxygen to the chamber. Once anesthetized, an appropriate size NLM was inserted by the Principal Investigator using a routine technique. The mask cuff was, then, inflated by injection of $3 \mathrm{~mL}$ of air into the cuff, and the piglets' respirations were assisted with a self-inflating bag to deliver intermittent positive pressure ventilation (IPPV). Further evidence of proper placement of the NLM was confirmed by auscultation and identifying breath sounds in all lung fields.

Piglets were monitored and hand bagged with self-inflating bags for 10 minutes. Heart rate, core temperature, $\mathrm{SaO}_{2}$, and $\mathrm{EtCO}_{2}$ were recorded continuously and charted minute-by-minute. After 10 minutes of monitoring piglets on the NLM, a customized closed suction system with a 6 French size (FR) catheter was attached to NLM with a v-adapter. (Kim-Vent, multi access catheter by Kimberly-Clark. Roswell, GA). Following its attachment, the catheter was advanced through the NLM for a pre-determined distance to establish the catheter tip beyond the NLM cuff boundary and into the trachea.
In order to examine the efficacy of the NLM in facilitating surfactant administration to the lungs, we simulated this process in the piglets by administering methylene blue through the attached catheter into the lungs. Specifically, $5 \mathrm{~mL}$ of normal saline mixed with methylene blue was injected in 2 aliquots into the trachea through the catheter tube. Each aliquot was followed by gentle positive-pressure assistance from the self-inflating bags, with the piglets rotated to another position prior to the subsequent aliquot. Postmortem autopsy was conducted to examine piglet trachea and lungs to determine the extent of spread of methylene blue.

\section{Specified outcomes}

Primary outcome measures were defined as successful insertion and placement of the NLM, control of the airways in the subjects, and the ability to maintain normal vital signs, core body temperature, heart rate, $\mathrm{SaO}_{2}$, and $\mathrm{EtCO}_{2}$ following successful NLM placement. Secondary outcome measures were constituted as the failure to the NLM after third attempt and fraction of inspired oxygen $\left(\mathrm{FiO}_{2}\right)$ exceeding $65 \%$ for 10 minutes to achieve a consistent oxygen saturation of $88-92 \%$.

\section{Sample size}

Based on a p-value equivalent to 0.05 , a sample size of 6 piglets was used for this pilot safety and efficacy trial to provide a power of $50 \%$.

\section{Statistical analysis}

Statistical analysis was primarily descriptive and exploratory in nature. We used piglets as an animal experiment model to establish the plausibility of an investigational medical device, and a $50 \%$ change would be considered statistically significant. Data were analyzed using the Mann-Whitney $\mathrm{U}$ test to compare the two groups of piglets both pre- and post-NLM insertion and examine the effects on physiological parameters between the two samples, with $\mathrm{P}<.05$ considered statistically significant.

\section{Results}

The NLM effectively controlled the piglet airways for the entire duration of the study and there were not any statistically significant differences in maintaining normal physiological parameters before and after insertion. Heart rate remained stable throughout the ventilation period in all the piglets and the average heart rate of the piglets during the 10-minute interval under NLM placement 
was comparable to the average heart rate of the piglets prior to insertion (Figure $2 \mathrm{~A}, \mathrm{U}$-value $14, \mathrm{p}=0.416$ ). Likewise, core body temperature remained stable throughout the ventilation period, with comparable average temperatures presented both during intervals with and without the insertion of the NLM (Figure 2B, U-value 18, $\mathrm{p}=0.468$ ). Piglet $\mathrm{SaO}_{2}$ under NLM placement was also stable and presented a comparable average across the 10-minute ventilation interval compared to ventilation mediated by hand bagging (Figure 2C, U-value 14, $\mathrm{p}=0.315$ ). The corresponding piglet $\mathrm{EtCO}_{2}$ was also stable throughout the ventilation period, with the average $\mathrm{EtCO}_{2}$ pre- and post-NLM insertion displaying, again, comparable levels (Figure 2D, U-value 11, $\mathrm{p}=0.416$ ) (Figure 2).
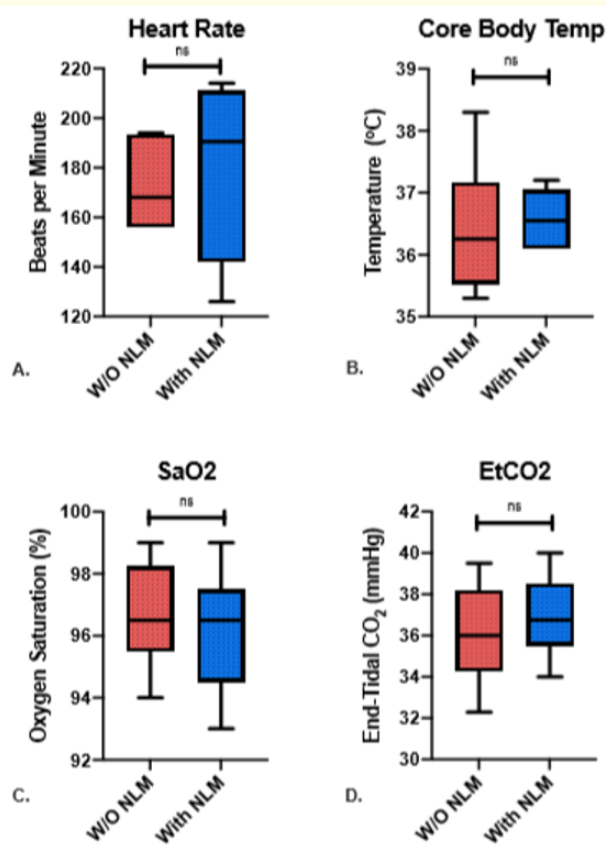

Figure 2: Piglet vital signs following administration of anesthesia and surfactant while using the NLM to maintain the airway. Vital signs were recorded over a ten-minute period during which the NLM was utilized to maintain the airway following the administration of anesthesia. There were no significant differences reported for heart rate $(A, U$-value $14, p=0.416)$, core body temperature (B, U-value 18, $\mathrm{p}=0.468$ ), $\mathrm{SaO} 2$ (C, U-value 14, $\mathrm{p}=$ 0.315 ), and EtCO2 (D, U-value 11, $p=0.416$ ) for the average recordings between the absence and use of the NLM. This suggests that the NLM can maintain a patent airway without incurring the same technicalities as needed during manual endotracheal intubation, as this requires the presence of many skilled personnel whereas the NLM can be placed without such constraints.
Regarding its safety, NLM placement was successful on first attempt and postmortem examination of trachea and lungs confirmed wide extent of spread of methylene Blue throughout the airways of all piglets. Dissection revealed proper placement of the NLM cuff at the opening of the glottis with corresponding catheter navigation through the NLM into the trachea (Figure 3). Further examination of the trachea and lungs revealed a consistent distribution of methylene blue spanning from the inner surface of the trachea through the alveoli as seen from the external borders of each apex (Figure 4). Additionally, there were no events of hypoxemia, bradycardia and any other unwanted effect during and after the insertion.

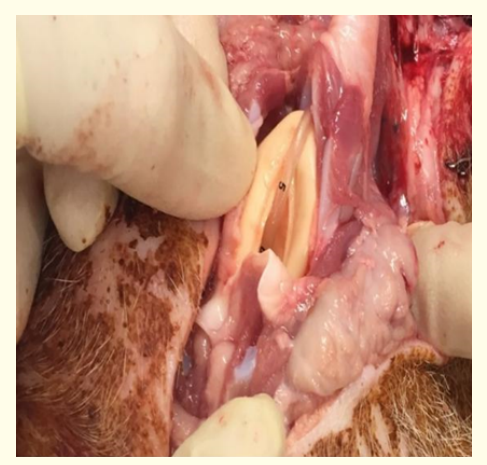

A

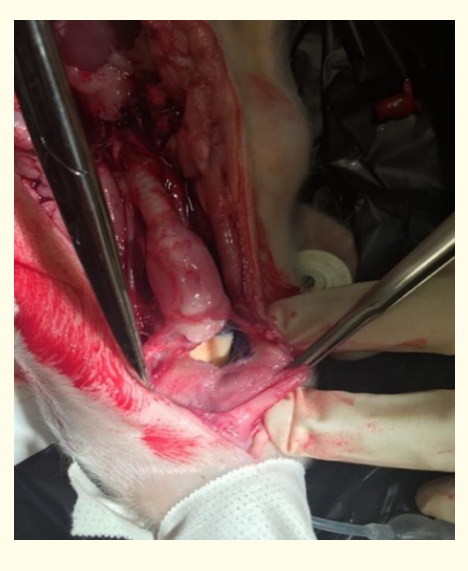

B

Figure 3: The NLM in situ with the tip of catheter passing into Trachea.

During postmortem examination, the NLM cuff is seen to be placed at the opening of the Glottis, which allows for the passing of a catheter through the opening into the trachea (A) and the continuation of the catheter to the remaining portion of the airway for methylene blue administration (B). 

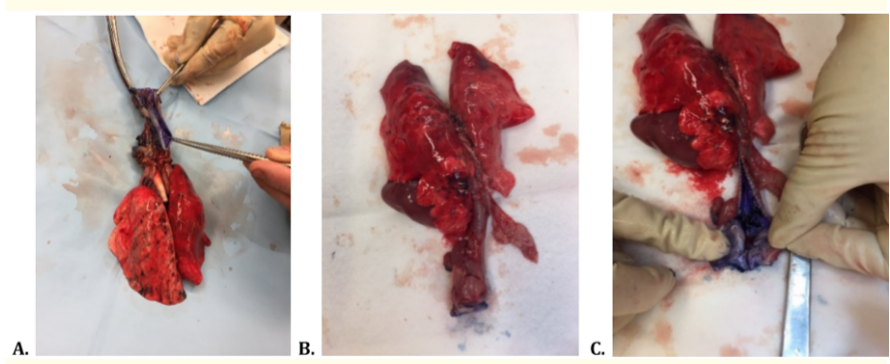

Figure 4: Postmortem examination of Piglet lungs and trachea showing staining of tracheal lining and lungs infiltration with methylene blue.

Postmortem examination of the airway (A) revealed staining from methylene blue, which was used to represent surfactant as administered using the NLM. Staining was observed from the exterior of the lungs (B), indicative of its penetration to the alveoli, as well as throughout the entirety of the internal surface area of the trachea (C).

\section{Discussion}

Modern developments in medical technology have created less strenuous means of maintaining airways during general anesthesia. However, these devices have failed to demonstrate the ability to effectively manage airways in premature infants due to respective size limitations. We designed a new device-the NLM-that can both ventilate and administer medication into the lungs of premature infants as well as full term babies and examined overall device efficacy and safety in managing airways while inducing piglets under general anesthesia.

Our results indicate that the insertion of NLM allows for safe and effective ventilation during anesthesia while maintaining a patent airway in piglets. Following the close monitoring of piglets under general anesthesia for 10 minutes both before and after insertion of the NLM, we found comparable heart rate, core body temperature, $\mathrm{SaO}_{2}$ and $\mathrm{EtCO}_{2}$ levels that lacked significant differences with $p$-values $>0.05$. Moreover, we demonstrated a $100 \%$ success rate of insertion during initial placement and administration of methylene Blue through the device to encompass the entire airway from the glottis and trachea through the alveoli. This was all accomplished without sustaining adverse effects or any other compromise to the piglet airways.
These findings suggest that the NLM upholds safety and efficacy standards that are in compliance with current ventilation techniques used on premature and full-term babies. Likewise, the findings associated with the successful administration of methylene blue suggest that the NLM possesses the ability to effectively administer surfactant to the patient in addition to managing the open airway. The consistent outcomes with respect to the recorded metrics before and after NLM insertion strongly present the NLM as a viable alternative to current airway management practices $[3,10]$.

Moreover, the consistent success of NLM insertion during the initial attempt for each piglet is indicative of a strong safety standard held by the device, and likely supports the hypothesis that the structure and design of the NLM mitigates the high dependence on skilled personnel for traditional endotracheal intubation. More near-term babies are being delivered at the local and community hospitals where neonatal services are not available and skills for endotracheal intubation procedure are lacking $[3,11]$. Despite excellent perinatal interventions including perinatal steroid administrations to mothers, 5 - 10\% babies born prematurely may require neonatal resuscitation and some would require airway control at birth [11]. The findings of this study are therefore especially compelling for use in such rural community hospitals.

Broadly, this data suggests that further studies in human neonates is justified using the NLM as an investigational medical device. If proven effective, some infants with respiratory distress syndrome may be able to receive airway control while avoiding intubation.

\section{Conclusion}

This study demonstrated the efficacy and safety of the NLM in piglets for ventilation and airway management under general anesthesia. While currently there is no appropriately sized laryngeal mask device available for management of ventilation and airway control in small laboratory animals during anesthesia, the NLM has shown promising results in piglets in efficacy as well as safety when compared to current practices in similarly sized populations. Further studies are necessary to test the NLM as an investigational medical device in human subjects to determine its viability in more representative clinical settings.

\section{Acknowledgements}

Author acknowledges Dr. William Keenan for reviewing the manuscript as well as his technical advice. 


\section{Conflict of Interest}

Author is the co-inventor of this prototype device and plans to use as an investigational medical device for human trials. Author has no financial conflict of interest with any medical device company to disclose.

\section{Bibliography}

1. Alanazi A. "Intubations and airway management: An overview of Hassles through third millennium". Journal of Emergencies, Trauma, and Shock 8.2 (2015): 99-107.

2. Reuter S., et al. "Respiratory distress in the newborn". Pediatrics in Review 35.10 (2014): 417-429.

3. Kribs A., et al. "Nonintubated surfactant application vs conventional therapy in extremely preterm infants: a randomized clinical trial". JAMA Pediatrics 169 (2015): 723-730.

4. Brain AI. "The development of the Laryngeal Mask--a brief history of the invention, early clinical studies and experimental work from which the Laryngeal Mask evolved". The European Journal of Anaesthesiology 4 (1991): 5-17.

5. Wemyss-Holden SA., et al. "The laryngeal mask airway in experimental pig anaesthesia". Laboratory Animals: SAGE Journals 33.1 (1999): 30-34.

6. Kazakos GM., et al. "Use of the laryngeal mask airway in rabbits: placement and efficacy". Laboratory Animals: SAGE Journals 36.4 (2007): 29-34.

7. M Fujita., et al. "Use of laryngeal mask airway in small animals". Journal of Veterinary Medical Science (1991): 531082.

8. Wanous AA., et al. "Feasibility of Laryngeal Mask Airway Device Placement in Neonates”. Neonatology 111.3 (2017): 222227.

9. Park C., et al. "The laryngeal mask airway in infants and children". The Canadian Journal of Anesthesia 48.4 (2001): 413417.
10. Mohammadizadeh M., et al. "Early administration of surfactant via a thin intratracheal catheter in preterm infants with respiratory distress syndrome: feasibility and outcome". The Journal of Pharmacy Practice and Research 4.1 (2015): 31-36.

11. Hillman $\mathrm{N}$ and Jobe AH. "Noninvasive strategies for management of respiratory problems in neonates". Neo Reviews 14 (2013): e227-e236.

\section{Volume 4 Issue 10 October 2021 \\ (C) All rights are reserved by Ayoob Ali.}

